\title{
Experimental evidence for a surface distribution of two-level systems in superconducting lithographed microwave resonators
}

\author{
Jiansong Gao, ${ }^{1, a)}$ Miguel Daal, ${ }^{2}$ Anastasios Vayonakis, ${ }^{1}$ Shwetank Kumar, ${ }^{1}$ \\ Jonas Zmuidzinas, ${ }^{1}$ Bernard Sadoulet, ${ }^{2}$ Benjamin A. Mazin, ${ }^{3}$ Peter K. Day, ${ }^{3}$ and \\ Henry G. Leduc ${ }^{3}$ \\ ${ }^{1}$ Division of Physics, Mathematics, and Astronomy, California Institute of Technology, Pasadena, California \\ 91125, USA \\ ${ }^{2}$ Physics Department, University of California at Berkeley, Berkeley, California 94720, USA \\ ${ }^{3}$ Jet Propulsion Laboratory, California Institute of Technology, Pasadena, California 91109, USA
}

(Received 29 February 2008; accepted 17 March 2008; published online 17 April 2008)

\begin{abstract}
We present measurements of the temperature-dependent frequency shift of five niobium superconducting coplanar waveguide microresonators with center strip widths ranging from 3 to $50 \mu \mathrm{m}$, taken at temperatures in the range of $100-800 \mathrm{mK}$, far below the $9.2 \mathrm{~K}$ transition temperature of niobium. These data agree well with the two-level system (TLS) theory. Fits to this theory provide information on the number of TLSs that interact with each resonator geometry. The geometrical scaling indicates a surface distribution of TLSs and the data are consistent with a TLS surface layer thickness of the order of a few nanometers, as might be expected for a native oxide layer. (C) 2008 American Institute of Physics. [DOI: 10.1063/1.2906373]
\end{abstract}

Superconducting microresonators have attracted substantial interest for low temperature detector applications due to the possibility of large-scale microwave frequency multiplexing. ${ }^{1-7}$ Such resonators are also being used in quantum computing experiments ${ }^{8-10}$ and for sensing nanomechanical motion. ${ }^{11}$ We previously reported that excess frequency noise is universally observed in these resonators and suggested that two-level systems (TLSs) in dielectric materials ${ }^{14,15}$ may be responsible for this noise. ${ }^{12}$ TLS effects are also observed in superconducting qubits. ${ }^{9}$ The TLS hypothesis is strongly supported by the observed temperature dependence of the noise and also by the observation of temperature-dependent resonance frequency shifts that closely agree with the TLS theory. ${ }^{13}$ To make further progress, it is essential to constrain the location of the TLSs, to determine whether they exist in the bulk substrate or in surface layers, perhaps oxides on the exposed metal or substrate surfaces, or in the interface layers between the metal films and the substrate. In this paper, we provide direct experimental evidence for a surface distribution of TLSs.

TLSs are abundant in amorphous materials ${ }^{14,15}$ and have electric dipole moments that couple to the electric field $\vec{E}$ of our resonators. For microwave frequencies and at temperatures $T$ between $100 \mathrm{mK}$ and $1 \mathrm{~K}$, the resonant interaction dominates over relaxation, which leads to a temperaturedependent variation of the dielectric constant given by ${ }^{16}$

$$
\frac{\Delta \epsilon}{\epsilon}=-\frac{2 \delta}{\pi}\left[\operatorname{Re} \Psi\left(\frac{1}{2}+\frac{1}{2 \pi i} \frac{\hbar \omega}{k T}\right)-\log \frac{\hbar \omega}{k T}\right],
$$

where $\omega$ is the frequency, $\Psi$ is the complex digamma function, and $\delta=\pi P d^{2} / 3 \epsilon$ represents the TLS-induced dielectric loss tangent at $T=0$ for weak nonsaturating fields. Here, $P$ and $d$ are the two-level density of states and dipole moment, as introduced by Phillips. ${ }^{16}$

Equation (1) has been extensively used to derive values of $P d^{2}$ in amorphous materials. If TLSs are present in super-

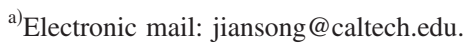

conducting microresonators, their contribution to the dielectric constant described by Eq. (1) could be observable as a temperature-dependent shift in the resonance frequency. Indeed, it has recently been suggested that the small anomalous low-temperature frequency shifts often observed in superconducting microresonators may be due to TLS effects, ${ }^{17,18}$ and, in fact, excellent fits to the TLS theory can be obtained. ${ }^{13}$ Assuming that the TLSs are uniformly distributed in a volume $V_{h}$ of a host material (e.g., a metal oxide or the bulk substrate), which has a dielectric constant of $\epsilon_{h}$, it can be shown that the fractional resonance frequency shift is given by

$$
\frac{\Delta f_{r}}{f_{r}}=-\frac{F}{2} \frac{\Delta \epsilon}{\epsilon},
$$

where the filling factor $F$ is given by

$$
F=\frac{\int_{V_{h}} \epsilon_{h}|\vec{E}(\vec{r})|^{2} d \vec{r}}{\int_{V} \epsilon|\vec{E}(\vec{r})|^{2} d \vec{r}}=\frac{w_{h}^{e}}{w^{e}} .
$$

The factor $F$ accounts for the fact that the TLS host material volume $V_{h}$ may only partially fill the resonator volume $V$, giving a reduced effect on the variation of resonance frequency. According to Eq. (3), $F$ is the ratio of the electric energy $w_{h}^{e}$ stored in the TLS-loaded volume to the total electric energy $w^{e}$ stored in the entire resonator.

The key idea of the experiment described in this paper is to measure $\Delta f_{r} / f_{r}$ of coplanar waveguide (CPW) resonators with different geometries in order to obtain values of $F \delta$ for each geometry. The frequency-multiplexed resonators are all simultaneously fabricated and integrated onto a single chip and are measured in a single cooldown. We can therefore safely assume that a single value of the loss tangent $\delta$ applies for all resonator geometries. This allows the variation of the filling factor $F$ with geometry to be determined, providing information on the geometrical distribution of the TLSs. If TLSs are in the bulk substrate with dielectric constant $\epsilon_{r}$, Eq. (3) applied to the CPW field distribution would yield a filling 


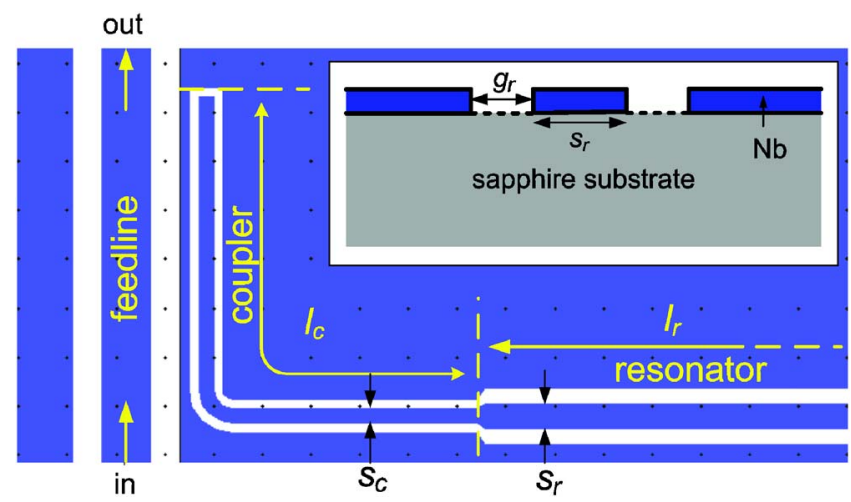

FIG. 1. (Color online) An illustration of the CPW coupler and resonator. The inset shows a cross-sectional view of the CPW. The contour of the metal surface and the contour of the exposed surface of the substrate are indicated by the solid line and the dashed line, respectively.

factor $F \approx \epsilon_{r} /\left(\epsilon_{r}+1\right)$ that is independent of the resonator's center strip width $s_{r}$. If instead the TLSs are in a surface layer, $F$ should be dependent on the CPW geometry, roughly scaling as $1 / s_{r}$.

We used a device with a 120-nm-thick Nb film deposited on a crystalline sapphire substrate, patterned into five CPW quarter-wavelength resonators with different geometries. Because $\mathrm{Nb}$ has a critical temperature of $T_{c}=9.2 \mathrm{~K}$, the effect of superconductivity on the temperature dependence of the resonance frequency is negligible for $T<1 \mathrm{~K}$. As shown in Fig. 1, each resonator is capacitively coupled to a common feedline using a CPW coupler with length of $l_{c} \cong 200 \mu \mathrm{m}$ and with a common center strip width of $s_{c}=3 \mu \mathrm{m}$. The coupler is then widened into the resonator body, with center strip widths of $s_{r}=3,5,10,20$, or $50 \mu \mathrm{m}$ and a length of $l_{r}$ $\sim 5 \mathrm{~mm}$. The ratio between the center strip width $s$ and the gap $g$ in both the coupler and the resonator body is fixed to $3: 2$ to maintain a constant impedance of $Z_{0} \approx 50 \Omega$. The resonance frequencies are $f_{r} \sim 6 \mathrm{GHz}$ and the coupler is designed to have a coupling quality factor of $Q_{c} \sim 50000$. The device is cooled in a dilution refrigerator, and its microwave output is amplified by using a cryogenic high electron mobility transistor (HEMT) amplifier on the $4 \mathrm{~K}$ stage. The complex transmission $S_{21}$ through the device and HEMT is measured by using a vector network analyzer locked to a rubidium frequency standard, and resonance frequencies are obtained by fitting these data. ${ }^{13}$

Figure 2 shows the measured frequency shifts $\Delta f_{r} / f_{r}$ for the five resonators as a function of temperature over the range of 100-800 $\mathrm{mK}$. Although all of the resonators display a common shape for the variation of frequency with temperature, the magnitude of the effect strongly varies with geometry. As shown by the dashed lines in Fig. 2, fits to the TLS model [Eq. (2)] generally agree quite well with the data. The nonmonotonic variation of the dielectric constant with temperature is familiar from the TLS literature: $f_{r}$ increases ( $\epsilon$ decreases) when $T>\hbar \omega / 2 k$; a minimum in $f_{r}$ (a maximum in $\epsilon$ ) occurs around $T=\hbar \omega / 2 k$; at lower temperatures $(T$ $<100 \mathrm{mK}$ ), and we would expect to see a decrease in $f_{r}$ (increase in $\epsilon$ ) as indicated by the extrapolation of the fit. The largest deviations from the TLS model (about 4\%) occur at the lowest temperatures and are likely due to TLS saturation effects. ${ }^{13}$ In this paper, we will ignore these small effects and focus on the geometrical dependence.

Downloaded 18 Apr 2008 to 131.215.225.137. Redistribution subject to AIP license or copyright; see http://apl.aip.org/apl/copyright.jsp

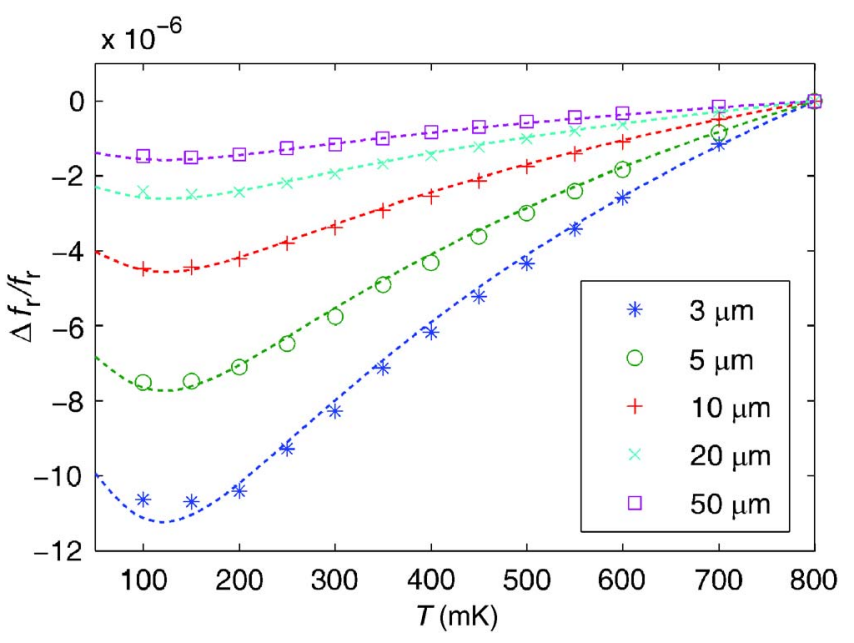

FIG. 2. (Color online) Fractional frequency shift $\Delta f_{r} / f_{r}$ as a function of temperature. $\Delta f_{r} / f_{r}$ is calculated by using $\Delta f_{r} / f_{r}=\left[f_{r}(T)-f_{r}(800 \mathrm{mK})\right] /$ $f_{r}(800 \mathrm{mK})$. The temperature sweep is in steps of $50 \mathrm{mK}$ from 100 to $600 \mathrm{mK}$ and in steps of $100 \mathrm{mK}$ above $600 \mathrm{mK}$. The markers represent different resonator geometries, as indicated by the values of the center strip width $s_{r}$ in the legend. The dashed lines indicate fits to the TLS theory [Eq. (2)].

For resonators that are wider than the coupler $\left(s_{r}>s_{c}\right)$, the measured values of $F \delta$ from the fits have to be corrected. In the limit $\left(l_{c} \ll l_{r}\right)$, the coupler correction is given by $F^{*}$ $=\left(F-t F_{3 \mu \mathrm{m}}\right) /(1-t)$, where $t=2 l_{c} /\left(l_{c}+l_{r}\right)$. The values of $F^{*} \delta$ are listed in Table I, as well as the ratios relative to the value for $3 \mu \mathrm{m}$ resonator.

A portion of the CPW inductance per unit length is contributed by the kinetic inductance of the superconductor. The kinetic inductance fraction $\alpha$ depends on CPW geometry and may be determined by measuring resonance frequency shifts at higher temperatures, closer to $T_{c} \cdot{ }^{19}$ We therefore measured the resonance frequencies at $4.2 \mathrm{~K}\left(0.46 T_{c}\right)$, allowing the shift $\Delta f_{r}(4.2 \mathrm{~K})=f_{r}(100 \mathrm{mK})-f_{r}(4.2 \mathrm{~K})$ as well as the kinetic inductance fraction to be calculated for each geometry, as shown in Table I.

Figure 3 shows the results for the geometrical scaling of the corrected filling factor $F^{*}$ and the kinetic inductance fraction $\alpha$, plotted as ratios relative to their respective values for the resonator with a $3 \mu \mathrm{m}$ wide center strip. The observed strong variation of $F^{*}$ with geometry immediately rules out a volume TLS distribution and favors a surface distribution. We investigate this further by comparing the data to two theoretically calculated geometrical factors $g_{m}$ and $g_{g}$, which have units of inverse length and are calculated from contour integrals in a cross-sectional plane given by $g_{m}$ $=\int_{\text {metal }} \vec{E}^{2} d l / V^{2}$ and $g_{g}=\int_{\text {gap }} \vec{E}^{2} d l / V^{2}$, where $V$ is the CPW

TABLE I. Values and ratios.

\begin{tabular}{|c|c|c|c|c|c|c|}
\hline $\begin{array}{l}s_{r} \\
(\mu \mathrm{m})\end{array}$ & $\begin{array}{c}\Delta f_{r} \\
(4.2 \mathrm{~K}) \\
(\mathrm{MHz})\end{array}$ & $\frac{\alpha}{\alpha_{3 \mu \mathrm{m}}}$ & $\begin{array}{c}F^{*} \delta \\
\left(\times 10^{-5}\right)\end{array}$ & $\frac{F^{*}}{F_{3 \mu \mathrm{m}}^{*}}$ & $\frac{g_{m}}{g_{m, 3 \mu \mathrm{m}}}$ & $\frac{g_{g}}{g_{g, 3 \mu \mathrm{m}}}$ \\
\hline 3 & 11.1 & 1 & $2.98 \pm 0.12$ & 1 & 1 & 1 \\
\hline 5 & 7.41 & 0.67 & $2.00 \pm 0.07$ & 0.67 & 0.62 & 0.64 \\
\hline 10 & 4.15 & 0.37 & $1.10 \pm 0.03$ & 0.37 & 0.33 & 0.35 \\
\hline 20 & 2.28 & 0.21 & $0.54 \pm 0.03$ & 0.18 & 0.17 & 0.19 \\
\hline 50 & 1.02 & 0.092 & $0.24 \pm 0.02$ & 0.08 & 0.075 & 0.086 \\
\hline
\end{tabular}




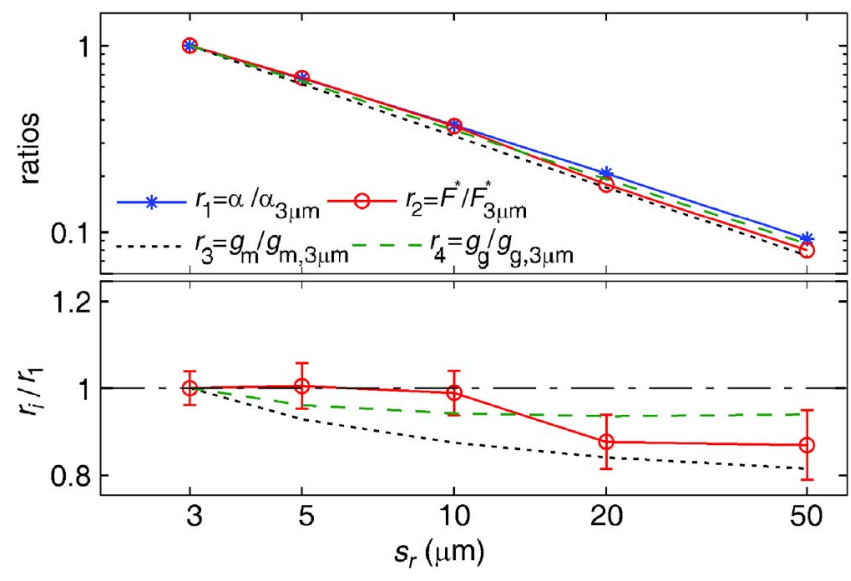

FIG. 3. (Color online) The scaling of the measured values of the kinetic inductance $\alpha$ and TLS filling factor $F^{*}$, as well as the calculated values of the CPW geometrical factors $g_{m}$ and $g_{g}$, are shown as a function of the resonator center strip width $s_{r}$. The top panel shows the ratios of $\alpha\left(r_{1}\right), F^{*}$ $\left(r_{2}\right), g_{m}\left(r_{3}\right)$, and $g_{g}\left(r_{4}\right)$ to their values for the $3 \mu \mathrm{m}$ resonators. The bottom panel shows these ratios normalized by the kinetic inductance ratio $r_{1}$.

voltage. The first integral is taken over the surfaces of the metal conductors and the second integral is taken over the exposed substrate surface, as illustrated in the inset of Fig. 1. The integrals are numerically evaluated by using the electric field derived from a numerical conformal mapping solution to the Laplace equation.

According to Eq. (3), $F^{*}$ should have the same scaling as $g_{m}$ if the TLSs are distributed on the metal surface (or at the metal-substrate interface) or as $g_{g}$ if the TLSs are located on the surface of the exposed substrate. The kinetic inductance of the CPW may also be calculated using a contour integral similar to that of $g_{m}$, except that the integrand is replaced by $\vec{H}^{2}{ }^{19}$ Because the magnetic field $\vec{H}$ is proportional to $\vec{E}$ for a CPW mode, we expect the kinetic inductance fraction $\alpha$ to have the same geometrical scaling as $g_{m}$.

Figure 3 shows that the four quantities, $F^{*}, \alpha, g_{m}$, and $g_{g}$, all scale as $s_{r}^{-\gamma}$ with $\gamma=0.85-0.91$. The finite thickness of the superconducting film is responsible for the deviations from $\gamma=1$. This is a very strong evidence that the TLSs have a surface distribution and are not uniformly distributed in the bulk substrate. Our data cannot discriminate between a TLS distribution on the metal surface from a TLS distribution on the exposed substrate surface (the gap) because the corresponding theoretical predictions $\left(g_{m}\right.$ and $\left.g_{g}\right)$ are very similar and both agree with the data. Future measurements of resonators with various center strip to gap ratios may allow these two TLS distributions to be separated.

The absolute values of $F^{*} \delta$ are also of interest. Assuming a typical value of $\delta \sim 10^{-2}$ for the TLS-loaded material, ${ }^{9}$ the measured value of $F^{*} \delta=3 \times 10^{-5}$ for the $3 \mu \mathrm{m}$ resonator yields a filling factor of $F^{*} \sim 0.3 \%$. Numerical calculations show that this is consistent with an $\sim 2$ nm layer of the TLSloaded material on the metal surface or an $\sim 3 \mathrm{~nm}$ layer on the gap surface, suggesting that native oxides or adsorbed layers may be the TLS host material.

In summary, the anomalous low-temperature frequency shifts of our superconducting CPW resonators are well explained by a model in which the TLSs are distributed on the surface of the CPW. The excess frequency noise ${ }^{2,12,13}$ also displays a strong geometrical dependence: for a fixed internal power, we find that the noise scales as $1 / s_{r}^{1.6}$, which is consistent with a surface distribution of TLS fluctuators. ${ }^{20}$ It therefore seems very likely that the TLSs, which cause anomalous low-temperature frequency shifts, are also responsible for the excess frequency noise. The use of optimized geometries or nonoxidizing materials may therefore offer a route to more sensitive photon detectors. The TLSs should also affect the resonator dissipation. At the relatively high power levels used in our experiments, TLS dissipation is strongly saturated ${ }^{9,12}$ and rather high values of the resonator quality factor $\left(Q_{r}>10^{5}\right)$ are routinely obtained. ${ }^{1}$ However, at low enough microwave power, the TLS response should become unsaturated, ${ }^{21}$ at which point the quality factor should be limited to $Q_{r} \sim 1 / F \delta$, or around $3 \times 10^{4}$ for the $3 \mu \mathrm{m}$ resonator described in this paper. This low-power regime is of direct relevance for quantum experiments, ${ }^{8}$ in which the microwave excitation of the resonator consists of one or a few photons.

We thank John Martinis, Clare Yu, and Sunil Golwala for useful discussions. The device was fabricated in the University of California, Berkeley, Microfabrication Laboratory. This work was supported, in part, by the NASA Science Mission Directorate, JPL, the Gordon and Betty Moore Foundation, and Alex Lidow, a Caltech Trustee.

${ }^{1}$ B. A. Mazin, P. K. Day, J. Zmuidzinas, and H. G. LeDuc, AIP Conf. Proc. 605, 309 (2002).

${ }^{2}$ P. K. Day, H. G. LeDuc, B. A. Mazin, A. Vayonakis, and J. Zmuidzinas, Nature (London) 425, 817 (2003).

${ }^{3}$ D. R. Schmidt, C. S. Yung, and A. N. Cleland, Appl. Phys. Lett. 83, 1002 (2003).

${ }^{4}$ J. A. B. Mates, G. C. Hilton, K. D. Irwin, L. R. Vale, and K. W. Lehnert, Appl. Phys. Lett. 92, 023514 (2008).

${ }^{5} \mathrm{G}$. Vardulakis, S. Withington, D. J. Goldie, and D. M. Glowacka, Meas. Sci. Technol. 19, 015509 (2008).

${ }^{6}$ J. Baselmans, S. J. C. Yates, R. Barends, Y. J. Y. Lankwarden, J. R. Gao, H. Hoevers, and T. M. Klapwijk, J. Low Temp. Phys. 151, 524 (2008).

${ }^{7}$ I. Hahn, P. K. Day, B. Bumble, and H. G. LeDuc, J. Low Temp. Phys. 151, 934 (2008).

${ }^{8}$ A. Wallraff, D. I. Schuster, A. Blais, L. Frunzio, R.-S. Huang, J. Majer, S. Kumar1, S. M. Girvin, and R. J. Schoelkopf, Nature (London) 431, 162 (2004).

${ }^{9}$ J. M. Martinis, K. B. Cooper, R. McDermott, M. Steffen, M. Ansmann, K. D. Osborn, K. Cicak, S. Oh, D. P. Pappas, R. W. Simmonds, and C. C. Yu, Phys. Rev. Lett. 95, 210503 (2005).

${ }^{10}$ M. A. Sillanpää, J. I. Park, and R. W. Simmonds, Nature (London) 449, 438 (2007).

${ }^{11}$ C. A. Regal, J. D. Teufel, and K. W. Lehnert e-print, arXiv:0801.1827.

${ }^{12}$ J. Gao, J. Zmuidzinas, B. A. Mazin, H. G. LeDuc, and P. K. Day, Appl. Phys. Lett. 90, 102507 (2007).

${ }^{13}$ S. Kumar, J. Gao, J. Zmuidzinas, B. A. Mazin, H. G. Leduc, and P. K. Day, Appl. Phys. Lett. 92, 123503 (2008).

${ }^{14}$ W. A. Phillips, J. Low Temp. Phys. 7, 351 (1972).

${ }^{15}$ P. W. Anderson, B. I. Halperin, and C. M. Varma, Philos. Mag. 25, 1 (1972).

${ }^{16}$ W. A. Phillips, Rep. Prog. Phys. 50, 1657 (1987).

${ }^{17}$ S. Kumar, P. Day, H. LeDuc, B. Mazin, M. Eckart, J. Gao, and J. Zmuidzinas, APS March Meeting Abstract, 2006, (http://meetings.aps.org/link/ BAPS.2006.MAR.B38.2).

${ }^{18}$ R. Barends, J. J. A. Baselmans, J. N. Hovenier, J. R. Gao, S. J. C. Yates, T. M. Klapwijk, and H. F. C. Hoevers, IEEE Trans. Appl. Supercond. 17, 263 (2007).

${ }^{19}$ J. Gao, J. Zmuidzinas, B. A. Mazin, P. K. Day, and H. G. Leduc, Nucl. Instrum. Methods Phys. Res. A 559, 585 (2006).

${ }^{20}$ J. Gao, M. Daal, B. A. Mazin, P. K. Day, H. G. Leduc, B. Sadoulet, and J. Zmuidzinas, e-print, arXiv:0804.0467.

${ }^{21}$ A. D. O'Connell, M. Ansmann, R. C. Bialczak, M. Hofheinz, N. Katz, E. Lucero, C. McKenney, M. Neeley, H. Wang, E. M. Weig, and A. N. Cleland, and J. M. Martinis, Appl. Phys. Lett. 92, 112903 (2008). 